\title{
Implementation of an enhanced recovery after thoracic surgery care pathway for thoracotomy patients-achieving better pain control with less (schedule II) opioid utilization
}

\author{
Karishma Kodia $^{\#}$, Joy A. Stephens-McDonnough ${ }^{\#}$, Ahmed Alnajar, Nestor R. Villamizar, Dao M. Nguyen \\ Thoracic Surgery Section, Division of Cardiothoracic Surgery, The DeWitt Daughtry Department of Surgery, Miller School of Medicine, University \\ of Miami, Miami, FL, USA \\ Contributions: (I) Conception and design: DM Nguyen; (II) Administrative support: JA Stephen-McDonough; (III) Provision of study materials or \\ patients: NR Villamizar, DM Nguyen, JA Stephen-McDonough; (IV) Collection and assembly of data: K Kodia, JA Stephen-McDonough; (V) Data \\ analysis and interpretation: A Alnajar, K Kodia, DM Nguyen; (VI) Manuscript writing: All authors; (VII) Final approval of manuscript: All authors. \\ \#These authors contributed equally to this work. \\ Correspondence to: Dao M. Nguyen, MD, MSc, FRCSC, FACS. Thoracic Surgery Section, Division of Cardiothoracic Surgery, The DeWitt Daughtry \\ Department of Surgery, Miller School of Medicine, University of Miami, Miami, FL, USA. Email: Dnguyen4@med.miami.edu.
}

Background: Enhanced recovery after surgery protocols incorporate evidence-based practices of pre-, intra- and post-operative care to achieve the most optimal surgical outcome, safe on-time discharge, and surgical cost efficiency. Such protocols have been adapted for specialty-specific needs and are implemented by a variety of surgical disciplines including general thoracic surgery. This study aims to evaluate the impact of our enhanced recovery after thoracic surgery (ERATS) protocol on postoperative outcomes, pain, and opioid utilization following thoracotomy.

Methods: This is a retrospective analysis of patients undergoing elective resection of intrathoracic neoplasms via posterolateral thoracotomy between $1 / 1 / 2016$ and 3/1/2020. Our enhanced recovery protocol, with a focus on multimodal pain management (opioid-sparing analgesics, infiltration of local anesthetics into intercostal spaces and surgical wounds, and elimination of thoracic epidural analgesia) was initiated on 2/1/2018. Demographics, clinicopathology data, subjective pain levels, peri-operative outcomes, in-hospital and post-discharge opioid utilization were obtained from the electronic medical record.

Results: A total of 98 patients (43 pre- and 55 post-protocol implementation) were included in this study. There was no difference in perioperative outcomes or percentage of opioid utilization between the two cohorts. The enhanced recovery group had significantly less acute pain. A significant reduction of in-hospital potent schedule II opioid use was noted following ERATS implementation [average MME: 10.5 (3.5-16.5) (ERATS) vs. 19.5 (12.6-36.0) (pre-ERATS), $\mathrm{P}<0.0001]$. More importantly, a drastic reduction of total and schedule II opioids dispensed at discharge was noted in the ERATS group [total MME: 150 (100.0-330.0) vs. 800.0 (450.0-975.0), $\mathrm{P}<0.0001$ and schedule II MME: 90.0 (0-242.2) vs. 800.0 (450.0-975.0), $\mathrm{P}<0.0001$; ERATS $v s$. pre-ERATS respectively]. A shorter hospital stay (median difference of 1 day, $\mathrm{P}=0.0012$ and a mean difference of 2.4 days, $\mathrm{P}=0.0054$ ) was observed in the enhanced recovery group.

Conclusions: Implementation of an enhanced recovery protocol for thoracotomy patients is safe and associated with elimination of thoracic epidural analgesia, decreased postoperative pain, shorter hospitalization, drastic reduction of post-discharge opioid dispensed and decreased dependence on addiction-prone schedule II narcotics.

Keywords: Thoracotomy; post-operative pain; post-operative opioid utilization; enhanced recovery after thoracic surgery (ERATS)

Submitted Mar 29, 2021. Accepted for publication May 20, 2021.

doi: $10.21037 /$ jtd-21-552

View this article at: http://dx.doi.org/10.21037/jtd-21-552

\footnotetext{
^ ORCID: 0000-0003-4807-7413.

ㄷ Journal of Thoracic Disease. All rights reserved.
} 


\section{Introduction}

Enhanced recovery after thoracic surgery (ERATS) protocols have gained significant traction in the past 5 years (1-5). Keeping the essential components of ERAS ${ }^{\circledR}$, as initially described in early 2000's and with adaptations over time (6), ERATS protocols have been developed for thoracic surgical patients. ERATS incorporates all nuances associated with the care of patients undergoing intrathoracic procedures, either by thoracotomy or by minimally invasive thoracoscopic surgery (MITS: video-assisted or robotic thoracoscopy) (7). Patients undergoing thoracic surgery are frequently elderly individuals with significant cardiopulmonary and metabolic comorbidities (8). Postoperative pain is intrinsic to thoracic surgical procedures, and pulmonary impairment following lung resections, together with underlying co-morbidities have a strong impact on post-operative outcomes. While the components of ERATS work synergistically to provide the most optimal outcomes, effective thoracic pain control with an opioid-sparing strategy, coupled with posterior intercostal nerve blocks and surgical wound infiltration with long-acting local anesthetic preparation liposomal bupivacaine, plays an essential role. Since the initial reports by Rice and Mehran of the MD Anderson Cancer Center $(9,10)$ that described and popularized this technique within the context of ERATS, many medical centers, including our own, have described successful implementation of ERATS with significant improvement of a wide range of outcome metrics such as reduced pain and opioid requirements, postoperative complications, and postoperative hospital length of stay (LOS) (1,2,11-13).

Reduction of postoperative pain and opioid requirements in thoracic patients has been associated with decreased postoperative complications, particularly cardiopulmonary complications, and decreased hospital LOS in those undergoing thoracotomies for pulmonary resections $(1,2,11,12)$. It has been argued that MITS, in and of itself, is a component of ERATS (5) and it is hard to improve already optimal outcome metrics such as postoperative LOS of MITS. Within the context of a well-implemented ERATS program, Krebs and colleagues have demonstrated equivalent short-term outcomes (LOS, complication rates, postoperative pain scores) of patients undergoing pulmonary lobectomy either by MITS or thoracotomy approaches (11). The most important effect of ERATS is the ability to reduce both in-hospital and post-discharge opioid requirements while maintaining adequate pain control $(2,12,13)$. Exposure to potent opioids (those classified as schedule II by the FDA such as morphine, oxycodone, hydromorphone) increases the risk of dependency $(14,15)$ and if made available outside the hospital setting would increase the risk of inappropriate use, especially in the current setting of the opioid crisis in the United States (16-19).

We implemented ERATS at our medical center on $2 / 1 / 2018$ for all patients undergoing thoracic surgical procedures. This study aims to assess the safety of ERATS protocol implementation and the effect of ERATS on mitigating postoperative pain, reducing opioid requirements, and evaluating short-term clinical outcomes of patients undergoing lung resection by thoracotomy until 3/1/2020, using a comparable cohort of pre-ERATS patients as historical controls.

The study was conducted and reported in concordance with the Strengthening the Reporting of Observational Studies in Epidemiology STROBE reporting checklist (20) (available at http://dx.doi.org/10.21037/jtd-21-552).

\section{Methods}

\section{Patient population}

A retrospective analysis of data extracted from our prospectively maintained thoracic surgery database and the electronic medical record $\mathrm{EPIC}^{\circledR}$ of patients at University of Miami Hospital was performed following institutional review board approval with a waiver of patient consent requirement (IRB: 20180827). The study was conducted in accordance with the Declaration of Helsinki (as revised in 2013). Patients undergoing posterolateral thoracotomy between $01 / 01 / 2016$ and $3 / 1 / 2020$ were reviewed. All adult patients ( $\geq 18$ years old) undergoing same-day admit elective pulmonary procedures (anatomic resection-segmentectomy, simple or bronchoplastic sleeve lobectomy, bilobectomy, pneumonectomy or non-anatomic sublobar wedge resections, the majority of which included intrathoracic lymphadenectomy for oncologic indications) or mediastinal-pleural procedures (resection of mediastinal tumors-mainly complex thymic neoplasms or pleural pathology such as fibrous tumor of pleura) via serratus anerior- and latissimus dorsi-sparing postero-lateral thoracotomy approach for curative-intent resections of intrathoracic neoplasms were included. Patients in whom accurate assessment of postoperative pain and narcotic use was not feasible such as those who remained on endotracheal intubation/mechanical 
ventilation following thoracotomy and those with longterm opioid use for chronic pain (determined by clinical history of taking scheduled opioid analgesics for at least 2 months immediately preceding thoracic procedures) were excluded. Patients who had sternotomy, clamshell, or hemiclamshell incisions, radical pleurectomy for primary or secondary pleural cancers, chest wall resections, esophageal procedures, or pleuro-pulmonary decortication for empyema were also excluded. We implemented our ERATS protocol (Table S1) on 2/1/2018 for all thoracic surgical patients who underwent either robotic thoracoscopy or thoracotomy. The thoracotomy patient cohort prior to this date (pre-ERATS group: 1/1/2016 to $1 / 31 / 2018$ ) served as historical control for the ERATS patients (2/1/2018 to 3/1/2020).

\section{Development and Implementation of ERATS.}

Our ERATS care protocol (Table S1) encompasses all aspects of pre-, peri-, intra- and post-operative management of thoracic surgical patients, similar to previously published protocols (1-7). Prior to formal implementation of ERATS, many of these care components were part of our routine practice but not uniform amongst various providers. Thoracic epidural analgesia was the main component of acute postoperative pain control supplemented by oral or intravenous schedule II opioids (morphine, oxycodone, and hydromorphone) for breakthrough pain prior to ERATS. Thoracic epidural catheters were placed prior to the induction of anesthesia and managed by the regional anesthesia team. Epidural analgesia typically was initiated at the end of the case with baseline infusion of bupivacaine with or without an opioid such as fentanyl or hydromorphone. The initial settings of epidural analgesia were routinely a basal infusion rate titrated to achieve optimal pain control and to avoid hypotension and a patient-control administration (PCA) option. It was typically removed one day after discontinuation of the chest tube. Other non-opioid analgesics (acetaminophen, tramadol, ketorolac, and ibuprofen) were used sparingly. The exact quantity and formulations of opioids used varied greatly based on the patients' need and the providers' discretion. One critical element of our ERATS implementation is our multimodal opioid (schedule II)sparing pain management strategy coupled with the offlabel use of liposomal bupivacaine (LipoB) $\left(\right.$ Exparel $^{\circledR}$, Pacira Pharmaceuticals Inc., Parsippany, NJ) for a single-dose intercostal nerve block and surgical wound infiltration to achieve durable regional analgesia up to 4 days. Moreover, ERATS patients received scheduled administration of acetaminophen, gabapentin, ibuprofen, or ketorolac (in the absence of contraindications such as renal dysfunction, high sanguineous chest tube drainage in the immediate postoperative period or known intolerance to NSAIDs) and the schedule IV opioid tramadol. Nursing staff in the pre-operative clinic, post-anesthesia care unit and thoracic surgery unit, as well as the operating room anesthesia staff, all received proper in-service ERATS training prior to implementation. The thoracic surgery unit nursing staff was regularly provided extensive in-service training regarding all components of the postoperative care, pain assessment and administration of PRN opioid analgesics commensurate to patient-reported pain levels. For intraoperative regional analgesia, LipoB was diluted with $50 \mathrm{~mL}$ of injectable saline and the initial $30 \mathrm{~mL}$ was used to infiltrate the sub-dermis of the entire intended thoracotomy prior to skin incision, upon entrance into the pleural cavity and the remaining $40 \mathrm{~mL}$ of the mixture was used to infiltrate 9 intercostal spaces as well as the soft tissue at the posterior thoracotomy site above and below the incision, similar to the technique described by Mehran and colleagues under direct vision using a 21 gauge spinal needle $(9,10)$. The nursing staff routinely performed scheduled objective pain assessments using the visual analog pain scale and administered rescue opioid analgesics (as prescribed) at their discretion. During the initial 6-month transition period following the launch of our ERATS program, clinical compliance by all healthcare providers was closely monitored and enforced. We routinely evaluated in-hospital pain levels and opioids required by patients throughout the ERATS implementation phase and tailored our post-discharge opioids prescription practices over time.

\section{Data collection and outcome metric measurements}

\section{Outcomes}

Safety outcomes were overall postoperative complications and 90-day mortality. Efficacy outcomes were postoperative pain, hospital length of stay, and in-hospital and post-discharge opioid utilization.

\section{Data source and description}

The thoracic surgery database prospectively collects detailed clinical parameters, including but not limiting to patient demographics, comorbidities, pulmonary function tests, body mass index (BMI), operative details, 
pathologic diagnoses, AJCC $8^{\text {th }}$ edition pathologic TNM staging for primary lung cancer, 90-day postoperative complications (using the Clavien-Dindo classification) (21) and hospital LOS in days, as well as postoperative readmission incidence. The database is maintained by a nurse practitioner and regularly audited for accuracy by the surgical faculty (DMN).

\section{Data attributes}

The following parameters were extracted from the hospital electronic medical record: patient-reported pain scores (scores were averaged over a 24-hour period and recorded up to 4 postoperative days), in-hospital analgesics dispensed (opioids: oxycodone, hydromorphone, morphine, fentanyl, tramadol; non-opioids: acetaminophen, gabapentin, ketorolac, ibuprofen, celecoxib, bupivacaine, lidocaine, liposomal bupivacaine). The quantities of opioids dispensed are expressed as p.o. morphine milligram equivalent (MME). Information regarding post-discharge re-admissions, either to our hospital or to another healthcare facility, were obtained from EPIC $^{\circledR}$ and also via post-discharge telephone follow-ups and clinic visits. Post-discharge analgesics including types and dosage of opioids prescribed were collected from the discharge summary. The filling and refilling (within 30-day after discharge) of all types of opioids were monitored by reviewing EPIC $^{\circledast}$ data and by routine surveying of our patients during telephone followups by our advanced registered nurse practitioner and by the attending surgeons at postoperative clinic visits. Such independently obtained information was frequently crossreferenced for accuracy. As EPIC ${ }^{\circledR}$ was implemented on $1 / 2017$ at our institution, reliable data on post-discharge opioid utilization were available only for 27 patients in the second half of the pre-ERATS period (1/1/2017 to $1 / 31 / 2018$ ).

\section{Statistical analysis}

Statistical analysis of categorical variables, expressed as percentages and frequencies, were analyzed using either Chi-square or Fisher's exact test as appropriate. Continuous variables were expressed as median and interquartile range and compared using a Student's $t$-test, an unequal variance $t$-test, or a Mann-Whitney $U$ test as appropriate (22). Univariable and multivariable linear, low-rank splines and logistic regression models were used to measure primary end-point outcomes. The low-rank regression splines were specified in the framework of generalized additive models and fitted to penalized likelihood estimation (GAMPL in SAS) to produce flexible nonparametric regressions and assess the relationship of ERATS on the total length of stay. The $95 \%$ confidence intervals were reported in the parametric models. In multivariable models, we adjusted for age, sex, and BMI. Mixed linear model test was used to analyze post-operative pain scores up to postoperative day 4 . We assumed linear time trends, giving rise to the intercept (initial pain at Day 0) and the slope (rate of change in pain per day on study) estimates. Then we used least squares means at each time point. Statistical analysis was performed with SAS software, version 9.4 (SAS Institute, Cary NC). A 2 -sided $\mathrm{P}$ value of $\leq 0.05$ denotes statistical significance.

\section{Results}

A total of 98 patients (43 in the pre-ERATS period and 55 after ERATS implementation) met the selection criteria and were included in our analysis. Patient demographics and clinical characteristics of the two cohorts were similar (Table 1). In patients with primary lung cancers, nearly $50 \%$ had locally advanced stage $3 \mathrm{~A} / \mathrm{B}$ or stage 4 cancer ( 3 cases due to unsuspected microscopic pleural metastasis). Similar numbers of patients in either cohort had induction chemotherapy for known pre-resection mediastinal N2 lymph node metastasis. All patients with primary lung cancer had anatomic lung resection and mediastinal lymphadenectomy. Similar incidences of other intrathoracic cancers (secondary lung cancers or other neoplasms: invasive thymoma, malignant solitary fibrous tumor of pleura) were noted in either group. There was no difference in estimated intraoperative blood loss or duration of operating room time between the two cohorts. All cases were staffed by either of the two attending surgeons (DMN, NV).

Regarding safety outcomes, there was no statistically significant difference in the overall incidence of complications (pre-ERATS: $37.2 \%$ vs. ERATS: $25.5 \%$ ) between the two groups $(\mathrm{P}>0.05)$ (Tables 2,3). Fewer pulmonary complications, however, were observed in ERATS patients. There was no 90-day mortality. Regarding the efficacy outcomes, ERATS was associated with a significant reduction of postoperative LOS (in days) [preERATS of $5.0(4.0-7.0)$ and 6.5 (4.3) vs. ERATS of 4.0 (3.0-6.0) and 4.4 (2.0), median (IQR) and mean (SD), $\mathrm{P}=0.0012$ and $\mathrm{P}=0.0054$ respectively] both in univariable (Estimate $=-0.375 ; \mathrm{P}<0.001$ ) and multivariable analysis $(-1.94 ; \mathrm{P}=0.003)$ which was adjusted for patient age, gender, 
Table 1 Demographics, clinical characteristics of all patients

\begin{tabular}{|c|c|c|c|c|}
\hline Patient and operative characteristics & Overall $(n=98)$ & Pre-ERATS $(n=43)$ & ERATS $(n=55)$ & $P$ value \\
\hline Age, median (IQR) & $68.0(58.5-73.5)$ & $68.0(60.0-73.5)$ & $65.0(57.0-73.2)$ & 0.3280 \\
\hline Gender (M:F) & $54: 44$ & $26: 17$ & $28: 27$ & 0.3453 \\
\hline ASA, median (IQR) & $3(3-3)$ & $3(3-3)$ & $3(3-3)$ & \\
\hline FEV1 (\% normal), median (IQR) & $74.0(60.5-87.5)$ & $68.5(55.2-82.7)$ & $74.5(62.7-93)$ & 0.40 \\
\hline DLCO (\% normal), median (IQR) & $72.5(62.0-84.5)$ & $70.0(62.0-79.0)$ & $73.0(60.0-85.7)$ & 0.08 \\
\hline \multicolumn{5}{|l|}{ Clinical characteristics } \\
\hline Primary lung cancer, n (\%) & $74(75.5)$ & $35(81.4)$ & $39(70.9)$ & 0.25 \\
\hline Induction therapy & $9(12.1)$ & $4(11.4)$ & $5(12.8)$ & \\
\hline $\begin{array}{l}\text { Secondary lung cancer and other } \\
\text { neoplasms, } n(\%)\end{array}$ & $24(25.5)$ & $8(18.6)$ & $16(29.1)$ & 0.25 \\
\hline Anatomic resections, n (\%) & $83(84.7)$ & $40(93.0)$ & $43(78.2)$ & 0.051 \\
\hline Wedge and other resections, n (\%) & $15(15.3)$ & $3(7.0)$ & $12(21.8)$ & \\
\hline Estimated blood loss (mL) & $100.0(80.0-175.0)$ & $100.0(100.0-200.0)$ & $100.0(100.0-200.0)$ & 0.82 \\
\hline Operating time (min) & $270.0(240.0-314.5)$ & $260.0(240.0-298.0)$ & $292.0(212.0-387.5)$ & 0.42 \\
\hline
\end{tabular}

ERATS, enhanced recovery after thoracic surgery; IQR, interquartile range; BMI, body mass index.

and BMI (Tables 2,3).

Patients in the ERATS group reported significantly less pain than the pre-ERATS cohort as shown in Figure 1 $(\mathrm{P}<0.0001)$. Despite the significant difference of baseline pain measurement (Estimate at time $0=-1.5021 ; \mathrm{P}=0.0003$ ) decreased patient-reported pain scores were significantly different between and within groups at 24, 48, 36, 64 hours after surgery: respective estimates $=-1.8635, \mathrm{P}<0.0001$; $-1.9009, \mathrm{P}<0.0001 ;-2.6871, \mathrm{P}<0.0001 ;-2.5093, \mathrm{P}<0.0001$. None of ERATS patients $v s .36$ of 43 pre-ERATS patients received thoracic epidural analgesia $(\mathrm{P}<0.00001)$. The other 7 pre-ERATS patients had other forms of postoperative pain management (patient-controlled analgesia with intravenous opioids and/or intercostal nerve blocks with local anesthetic agents) due to patient refusals or unsuccessful insertion of the catheter.

The use of acetaminophen and NSAIDs was similar between both groups while $95 \%$ of ERATS patients received gabapentin vs. $16 \%$ in pre-ERATS cohort $(\mathrm{P}<0.00001)$ (Table 4). In-hospital opioid utilization was similar in both groups even in the absence of thoracic epidural analgesia in the ERATS patients (Figure 2A). A 2 -fold decrease in schedule II opioid use was observed in ERATS patients [ERATS daily average MME median (IQR): 10.5 (3.5-16.9) vs. pre-ERATS daily average MME median (IQR): 19.5 (12.6-36.0), $\mathrm{P}<0.0001]$ (Figure 2A), which was coupled with more schedule IV opioid tramadol use for postoperative analgesia (Table 4, Figure 2A). More importantly, ERATS was associated with a 5 -fold reduction of post-discharge opioid prescribed [pre-ERATS total MME: 800.0 (450.0-975.0) vs. ERATS total MME: 150.0 (100.0-330.0), $\mathrm{P}<0.0001]$. Notably there was a 10 -fold reduction of schedule II opioids prescribed for patients of the ERATS cohort [pre-ERATS MME: 800.0 (450.0-975.0) vs. ERATS MME: $90.0(0-242.2), \mathrm{P}<0.0001]$. Similar to the in-hospital opioid utilization profile, more tramadol was prescribed at discharge for patients in the ERATS group (Figure 2B). There was a significant reduction in the incidence of both initial filling and re-filling of schedule II opioids after discharge in ERATS patients (Table 4). For instance, $78 \%$ of ERATS patients filled their schedule II initial prescription vs. $100 \%$ of pre-ERATS patients 
Table 2 Post-operative outcomes

\begin{tabular}{|c|c|c|c|}
\hline Post-operative outcome & Pre-ERATS $(n=43)$ & ERATS $(n=55)$ & $P$ value \\
\hline Hospital LOS [median (IQR); mean (SD)] & $5.0(4.0-7.0) ; 6.5(4.3)$ & $4.0(3.0-6.0) ; 4.4(2.0)$ & $0.0012 ; 0.0054$ \\
\hline \multicolumn{4}{|l|}{ Post-operative outcomes } \\
\hline \multicolumn{4}{|l|}{ Complications (Clavien-Dindo), n (\%) } \\
\hline $1-2$ & 8 & 9 & \\
\hline $3-4$ & 8 & 5 & \\
\hline 5 & 0 & 0 & \\
\hline Cardiovascular & 3 & 5 & 1.00 \\
\hline TEA-induced hypotension/ICU & 1 & 0 & \\
\hline Pulmonary & 12 & 6 & 0.038 \\
\hline Respiratory insufficiency requiring mechanical ventilation & 4 & 2 & \\
\hline Fiberoptic bronchoscopy for atelectasis/pneumonia & 4 & 2 & \\
\hline Bronchopleural fistula/empyema & 1 & 0 & \\
\hline $\begin{array}{l}\text { Chest tube re-insertion (pneumothorax/subcutaneous } \\
\text { emphysema) }\end{array}$ & 2 & 0 & \\
\hline Air-leak $>5$ days & 1 & 2 & \\
\hline Re-operations & 0 & 2 & 0.50 \\
\hline Re-admissions, n (\%) & $6(13.9)$ & $2(3.6)$ & 0.13 \\
\hline
\end{tabular}

\#, highest score of multiple complications. ERATS, enhanced recovery after thoracic surgery; LOS, length of stay; IQR, interquartile range; $\mathrm{SD}$, standard deviation; ICU, intensive care unit; FUO, fever of unknown origin.

$(\mathrm{P}=0.0065)$. Only $17.2 \%$ of ERATS patients needed a refill of their opioid prescription $v s .40 .7 \%$ of the pre-ERATS cohorts $(\mathrm{P}=0.034)$ and only $50 \%$ of those refills were schedule II $v s .100 \%$ of refills for pre-ERATS patients were scheduled II opioids ( $\mathrm{P}=0.012)$.

\section{Discussion}

Our retrospective analysis demonstrated that ERATS implementation for patients undergoing posterolateral thoracotomy for curative-intent resection of intrathoracic neoplasms was safe, efficacious, and associated with significantly less acute postoperative pain. It also leads to complete elimination of thoracic epidural analgesia alongside its potential adverse side effects $(23,24)$, without increasing in-hospital opioid requirements. Most importantly it allowed for a drastic reduction of postdischarge opioid utilization. A notable observation is 
Table 3 Univariate and multivariate analysis of outcomes including complications, length of stay and prolonged length of stay

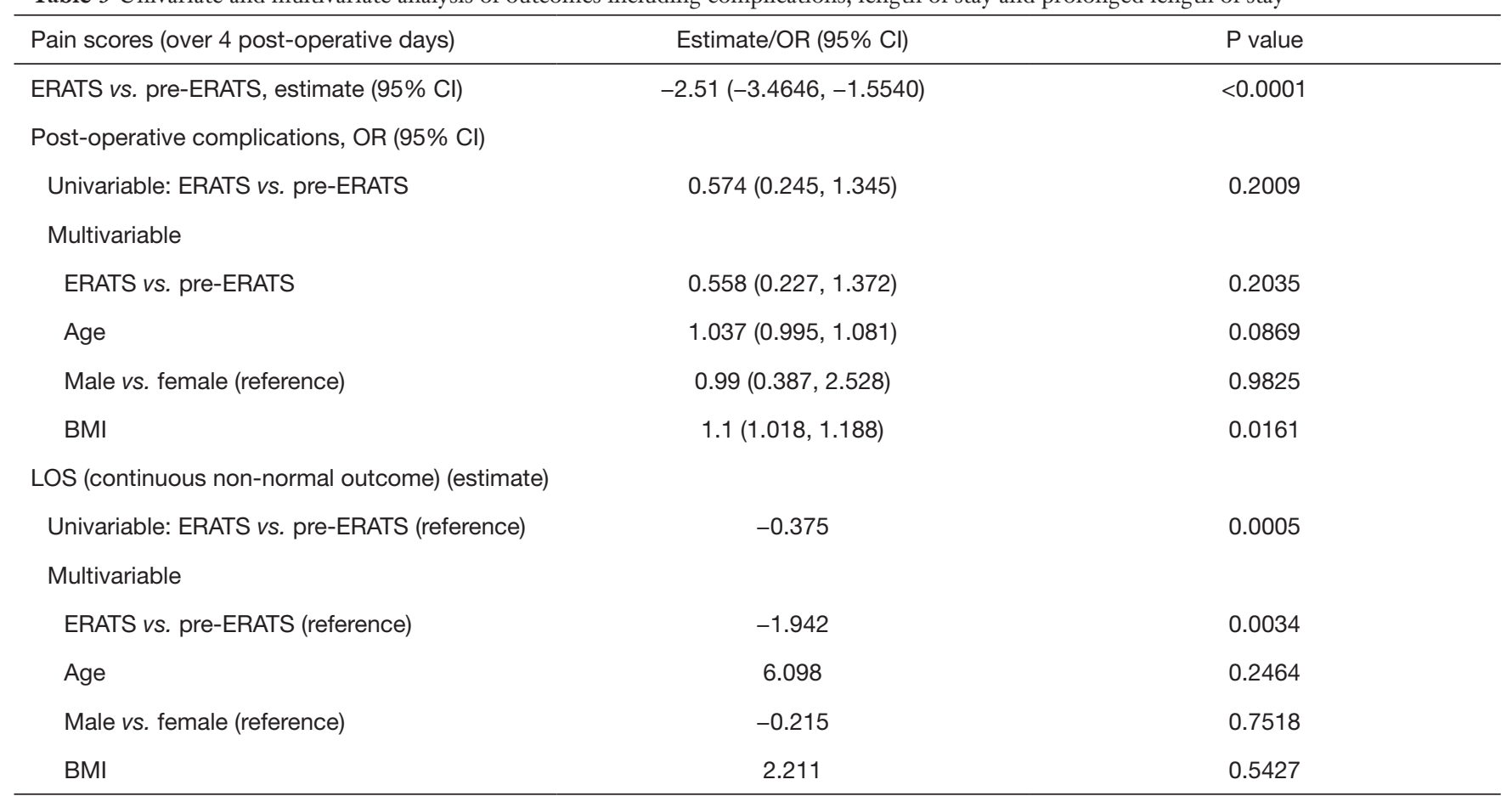

ERATS, enhanced recovery after thoracic surgery; LOS, length of stay; BMI, body mass index.

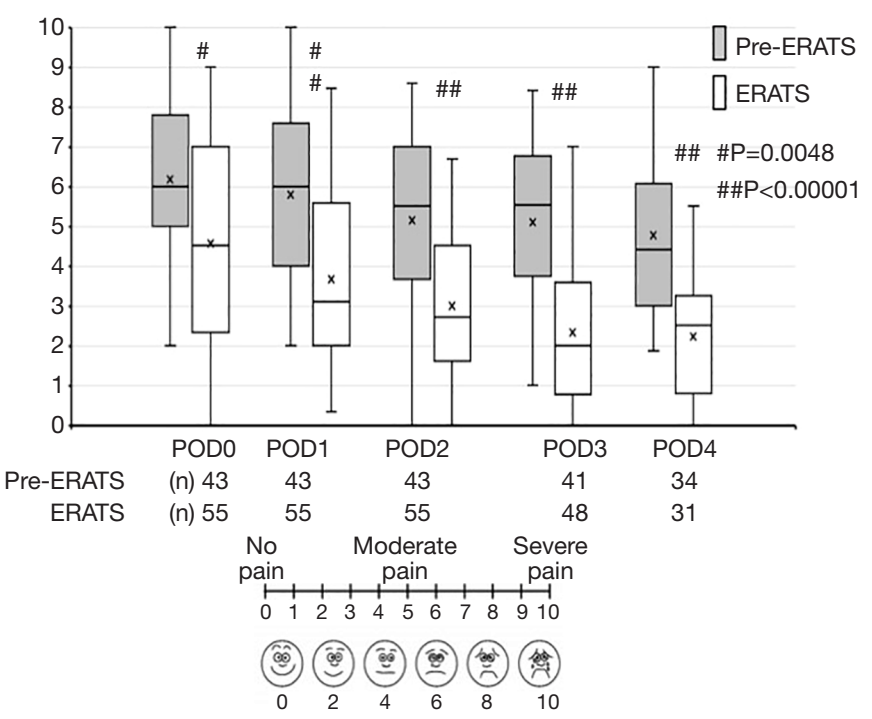

Figure 1 Significant reduction of postoperative pain in ERATS patients. Postoperative patient-reported subjective pain scores before and after implementation of ERATS in thoracotomy patients using the visual analog pain scale (0: no pain to 10: worst pain possible). Daily pain scores are expressed using the box-whisker plots (box: IQR, -: median, x: mean, minimal and maximal values and outliers) over multiple postoperative days (POD) for each group. $\mathrm{n}$ represents the number of subjects per group for that particular POD. A mixed effects model analysis revealed significant individual differences in post-operative pain trajectory slopes. 
Table 4 Post-thoracotomy in-hospital and post-discharge analgesic utilization

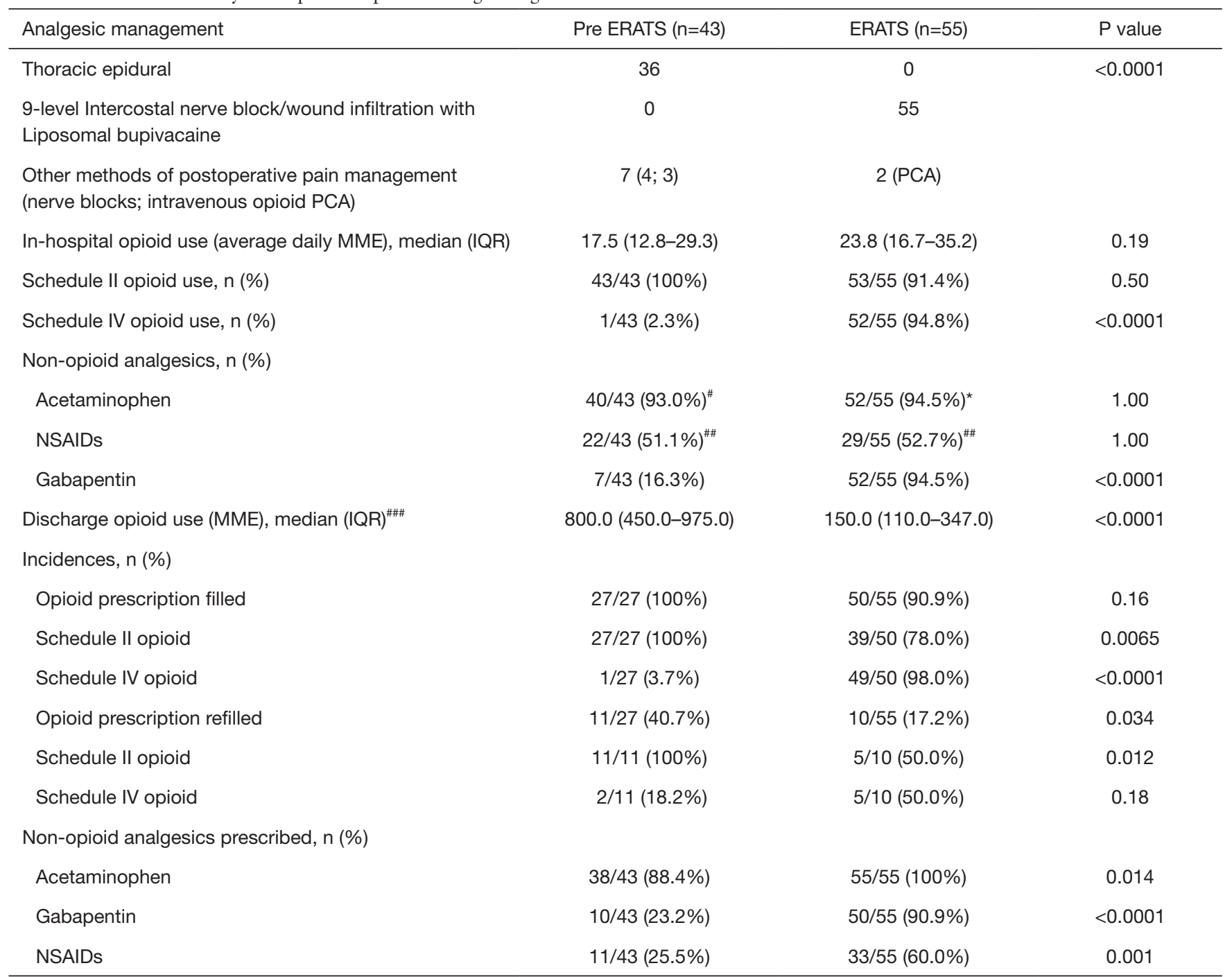

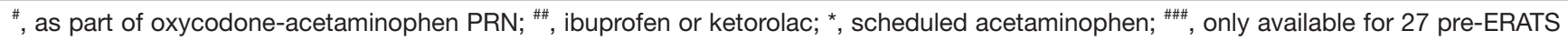
patients. ERATS, enhanced recovery after thoracic surgery; PCA, patient-controlled analgesia; MME, morphine milligram equivalent; IQR, interquartile range.

the ability of ERATS to decrease a reliance on schedule II opioids both in-hospital and after discharge for pain management. Our study also provided a granular quantitative analysis of post-discharge opioid utilization (total amount and subtypes of opioid prescribed and incidence of fill and refill of narcotic prescriptions), a feature not previously reported. Moreover, ERATS was also associated with a statistically significant reduction of hospital LOS in general and for prolonged LOS hospital courses.

Our study was able to shed light on the impact of ERATS for three main metrics: (I) clinical outcomes including hospital LOS, (II) patient-reported subjective pain levels and (III) opioid prescription (particularly schedule II narcotics) requirements. Before the formal implementation of ERATS at our institution, many prophylactic measures had already been instituted to minimize postoperative gastrointestinal, genitourinary, and cardiopulmonary complications. This resulted in little difference (except for a clear reduction of pulmonary adverse events) in the rate of postoperative complications between two patient cohorts. The ERATS patients however had a shorter hospital LOS that was attributable to better pain control, early ambulation, timely removal of tubes and drains, and patients 

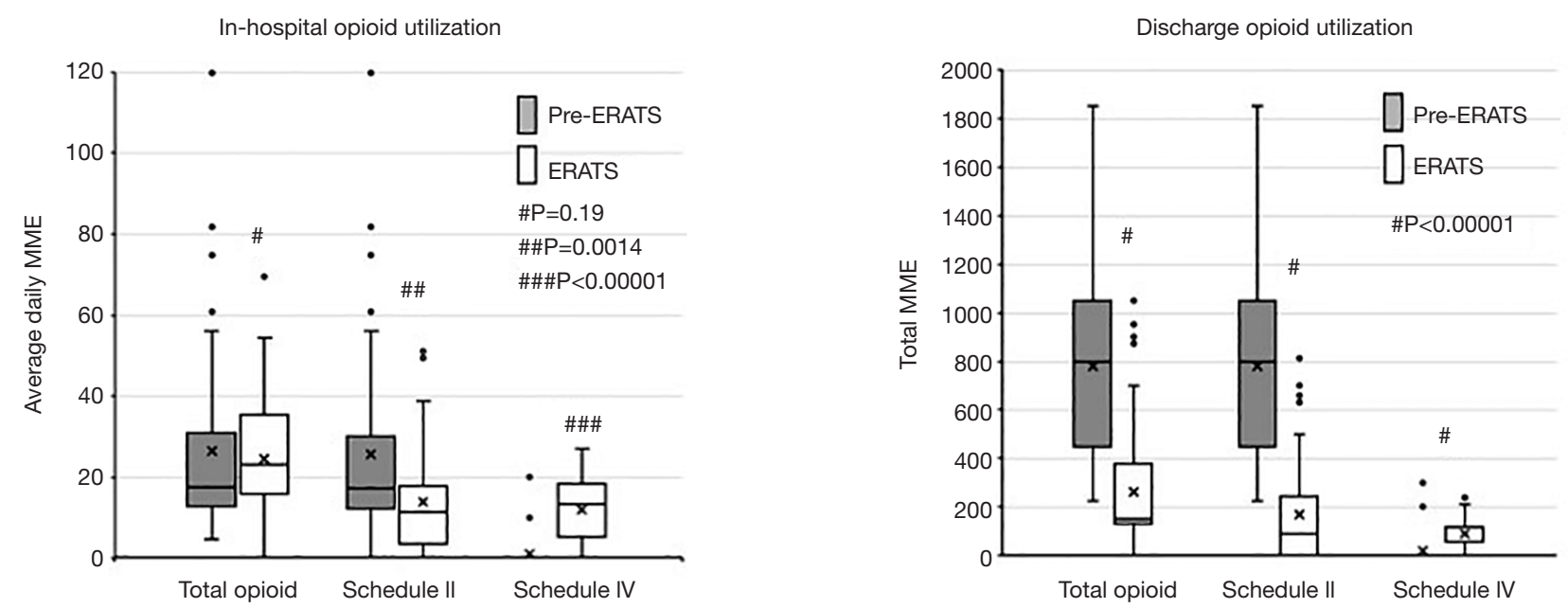

Figure 2 In-hospital and post-discharge opioid dispenses before and after implementation of ERATS in thoracotomy patients, expressed as average daily MME (calculated by dividing total MME of entire hospital stay with number of postoperative days in hospital) for in-hospital opioid use (A) and total MME for post discharge opioid prescription (B) using the box-whisker plots. While there was no difference in the total in-hospital opioid utilization between the two cohorts, a 2-fold reduction of schedule II opioid and increased tramadol use was seen in ERATS patients. There was a 5 -fold and 10-fold reduction of total opioid and schedule II opioid prescribed for ERATS patients at discharge from the hospital (B). Pairwise statistical analysis was performed using Mann-Whitney U test. ERATS, enhanced recovery after thoracic surgery; MME, morphine milligram equivalent.

clinically and psychologically ready to be discharged. Our findings recapitulated previously described salutary results of ERATS. Van Haren and colleagues from MD Anderson Cancer Center (Houston, TX) reported in a large patient cohort over an 11-year period (1/1/2006-12/31/2016) significant reduction of cardio-pulmonary complications and hospital LOS following ERATS implementation in thoracotomy patients (1). In a more contemporary dataset (2015 to 2017), Martin and colleagues from the University of Virginia (Charlottesville, VA) reported that ERATS was associated with a significant reduction of hospital LOS but no changes in post-thoracotomy complications (except for reduction of postoperative atelectasis) (2). Going beyond these initial observations, Krebs and colleagues further demonstrated that, after riskadjustment and within the context of a well-applied ERATS program, there was no difference in postoperative pain, opioid use, complication rates or hospital LOS between patients undergoing lobectomy for lung cancer either by thoracotomy or video-assisted thoracoscopy (11). Our own data presented here and previously published for ERATS in robotic thoracoscopic patients (13) certainly supports this notion even though we have not formally performed such comparative analysis. Since its establishment, our ERATS protocol for thoracotomy has served our patients well.
We noticed that safe elimination of the use of thoracic epidural analgesia simplified care for these thoracotomy patients. The surgical team now has total control of the pain management with real-time assessment of postoperative pain level and can effectively address patients' analgesic needs. The highlight of ERATS is the combination of intercostal nerve blocks with liposomal bupivacaine, multiple non-opioid analgesics of different mechanisms of action, and tramadol, a schedule IV opioid, to achieve effective pain control and to mitigate reliance on potent, addiction-prone schedule II opioids such as morphine, oxycodone, or hydromorphone. In fact, as shown in Figure 1, ERATS patients without thoracic epidural analgesia had significantly less pain than pre-ERATS patients. While being an effective analgesic, NSAIDs (either ketorolac or ibuprofen) could only be given in about $50 \%$ to $60 \%$ of our patients due to clinical or pharmacological contraindications. A significant amount of opioids prescribed at hospital discharge, particularly schedule II narcotics, in the pre-ERATS cohort reflected an outdated prescribing practice, which did not exploit the use of schedule IV opioid tramadol and non-opioid analgesics and also likely represented over-prescription. Pre-ERATS patients routinely received discharge prescriptions of 14 -day supply oxycodone/acetaminophen $(5 / 325 ; 1$ to 2 tablets 
Q6hrs PRN or 420 to 840 MME). ERATS patients were discharged with amounts and types of opioids based on their postoperative pain levels and in-hospital opioid needs together with all other non-opioid analgesics, achieving a much lower post-discharge MME similar to practice patterns for general surgical patients $(25,26)$ and thoracic patients (27). Over-prescription of opioids for postoperative patients is a well-recognized phenomenon $(18,25,26)$. This was mitigated following ERATS implementation. The low MME and reduced incidence of opioid (particularly schedule II) refills by patients indicated that their pain was adequately controlled and they were not denied of adequate opioid analgesics at the time of discharge from the hospital. Decreased early postoperative pain in ERATS patients correlated with fewer out-of-hospital opioid needs (a surrogate of pain levels), a notion compatible with the previous observation by Katz et al. in a landmark study in 1996 demonstrating early postoperative pain intensity was linked to development of persistent postoperative pain and possible persistent opioid use $(28,29)$. Our study focuses on acute post-thoracotomy pain and short-term use of opioids, particularly potent schedule II narcotics, and not on longterm persistent pain and chronic opioid use following after thoracotomy. Reduction of postoperative schedule II opioid use has many salutary effects including decreased drug exposure leading to lower risk of dependence/addiction, less availability of opioids in the community and reducing the opportunity for inappropriate use/abuse particularly in this current opioid epidemic in the USA. To put this in a broader context, in a study by Chen et al. of 18,343 patients, $46 \%$ were prescribed opioids at hospital discharge. There was a trend of $36 \%$ over prescription in the thoracic service (30), demonstrating the concerning role of health care professionals in contributing to the opioid pandemic, particularly since the surplus medication following surgery is the primary source of prescription diversion. Thus, it is imperative to understand how we can limit opioid use within the healthcare system while providing optimal outcomes.

This study has many limitations. It is a single-institution retrospective longitudinal case series analysis of a beforeand-after nature that carries intrinsic biases. The study also suffers from the small sample size despite attempts to extend the study for more than four years around the ERATS initiation date. This population represented about half of our open cases over the study period. We perform most of our thoracic procedures for lung cancer using robot-assisted thoracoscopy and reserve a thoracotomy approach for complex lung resections. Another limitation is our inability to complete the post-discharge MME for the first half of the pre-ERATS cohort but there is no reason to believe that these patients would have been given prescriptions for a much different amount of opioids (higher or lower) at the time of discharge. These limitations, however, would not diminish the significance of our observations on the salutary effects of ERATS in thoracotomy patients.

In conclusion, this retrospective analysis highlights many positive impacts of ERATS implementation on patients undergoing thoracotomy for pulmonary resection. Notably there is a reduction of hospital LOS, postoperative pain and decreased reliance on opioids, particularly schedule II class of narcotics, for adequate acute postoperative pain management. This ERATS protocol is now the standard of care for our thoracic patients.

\section{Acknowledgments}

The University of Miami supported this work. Funding: None.

\section{Footnote}

Reporting Checklist: The authors have completed the STROBE reporting checklist. Available at http://dx.doi. org/10.21037/jtd-21-552

Data Sharing Statement: Available at http://dx.doi. org/10.21037/jtd-21-552

Peer Review File: Available at http://dx.doi.org/10.21037/jtd21-552

Conflicts of Interest: All authors have completed the ICMJE uniform disclosure form (available at http://dx.doi. org/10.21037/jtd-21-552). The authors have no conflicts of interest to declare.

Etbical Statement: The authors are accountable for all aspects of the work in ensuring that questions related to the accuracy or integrity of any part of the work are appropriately investigated and resolved. The study was performed following institutional review board approval with a waiver of patient consent requirement (IRB: 20180827). The study was conducted in accordance with the Declaration of Helsinki (as revised in 2013).

Open Access Statement: This is an Open Access article 
distributed in accordance with the Creative Commons Attribution-NonCommercial-NoDerivs 4.0 International License (CC BY-NC-ND 4.0), which permits the noncommercial replication and distribution of the article with the strict proviso that no changes or edits are made and the original work is properly cited (including links to both the formal publication through the relevant DOI and the license). See: https://creativecommons.org/licenses/by-nc-nd/4.0/.

\section{References}

1. Van Haren RM, Mehran RJ, Mena GE, et al. Enhanced Recovery Decreases Pulmonary and Cardiac Complications After Thoracotomy for Lung Cancer. Ann Thorac Surg 2018;106:272-9.

2. Martin LW, Sarosiek BM, Harrison MA, et al. Implementing a Thoracic Enhanced Recovery Program: Lessons Learned in the First Year. Ann Thorac Surg 2018;105:1597-604.

3. Madani A, Fiore JF Jr, Wang Y, et al. An enhanced recovery pathway reduces duration of stay and complications after open pulmonary lobectomy. Surgery 2015;158:899-908; discussion 908-10.

4. Haro GJ, Sheu B, Marcus SG, et al. Perioperative Lung Resection Outcomes After Implementation of a Multidisciplinary, Evidence-based Thoracic ERAS Program. Ann Surg 2019. [Epub ahead of print]. doi: 10.1097/SLA.0000000000003719.

5. Brunelli A, Thomas C, Dinesh P, Lumb A. Enhanced recovery pathway vs. standard care in patients undergoing video-assisted thoracoscopic lobectomy. J Thorac Cardiovasc Surg 2017;154:2084-90.

6. Ljungqvist O, Scott M, Fearon KC. Enhanced Recovery After Surgery: A Review. JAMA Surg 2017;152:292-8.

7. Batchelor TJP, Rasburn NJ, Abdelnour-Berchtold E, et al. Guidelines for enhanced recovery after lung surgery: recommendations of the Enhanced Recovery After Surgery (ERAS) Society and the European Society of Thoracic Surgeons (ESTS). Eur J Cardiothorac Surg 2019;55:91-115.

8. Broderick SR, Grau-Sepulveda M, Kosinski AS, et al. The Society of Thoracic Surgeons composite score rating for pulmonary resection for lung cancer. Ann Thorac Surg 2020;109:848-55.

9. Mehran RJ, Walsh GL, Zalpour A, et al. Intercostal Nerve Blocks With Liposomal Bupivacaine: Demonstration of Safety, and Potential Benefits. Semin Thorac Cardiovasc Surg 2017;29:531-37.
10. Rice DC, Cata JP, Mena GE, et al. Posterior Intercostal Nerve Block With Liposomal Bupivacaine: An Alternative to Thoracic Epidural Analgesia. Ann Thorac Surg 2015;99:1953-60.

11. Krebs ED, Mehaffey JH, Sarosiek BM, et al. Is less really more? Reexamining video-assisted thoracoscopic vs. open lobectomy in the setting of an enhanced recovery protocol. J Thorac Cardiovasc Surg 2020;159:284-94.

12. Rice D, Rodriguez-Restrepo A, Mena G, et al. Matched Pairs Comparison of an Enhanced Recovery Pathway vs. Conventional Management on Opioid Exposure and Pain Control in Patients Undergoing Lung Surgery. Ann Surg 2020. [Epub ahead of print]. doi: 10.1097/ SLA.0000000000003587.

13. Razi SS, Stephens-McDonnough JA, Haq S, et al. Significant reduction of postoperative pain and opioid analgesics requirement with an Enhanced Recovery After Thoracic Surgery protocol. J Thorac Cardiovasc Surg 2021;161:1689-701.

14. Clarke H, Soneji N, Ko DT, et al. Rates and risk factors for prolonged opioid use after major surgery: population based cohort study. BMJ 2014;348:g1251.

15. Brescia AA, Harrington CA, Mazurek AA, et al. Factors Associated With New Persistent Opioid Usage After Lung Resection. Ann Thorac Surg 2019;107:363-8.

16. Lee JS, Hu HM, Edelman AL, et al. New Persistent Opioid Use Among Patients With Cancer After CurativeIntent Surgery. J Clin Oncol 2017;35:4042-9.

17. Nelson DB, Niu J, Mitchell KG, et al. Persistent Opioid Use Among the Elderly After Lung Resection: A SEERMedicare Study. Ann Thorac Surg 2020;109:194-202.

18. Bicket MC, Long JJ, Pronovost PJ, et al. Prescription Opioid Analgesics Commonly Unused After Surgery: A Systematic Review. JAMA Surg 2017;152:1066-71.

19. Hurley R. US surgeon general: doctors have central role in solving opioid epidemic. BMJ 2017;356:j715.

20. von Elm E, Altman DG, Egger M, et al. STROBE Initiative. The Strengthening the Reporting of Observational Studies in Epidemiology (STROBE) Statement: guidelines for reporting observational studies. Int J Surg 2014;12:1495-9.

21. Dindo D, Demartines N, Clavien PA. Classification of surgical complications: a new proposal with evaluation in a cohort of 6336 patients and results of a survey. Ann Surg 2004;240:205-13.

22. Ruxton GD. The unequal variance $t$-test is an underused alternative to Student's t-test and the Mann-Whitney U test. Behav Ecol 2006;17:688-90. 
23. von Hösslin T, Imboden P, Lüthi A, Rozanski MJ, et al. Adverse events of postoperative thoracic epidural analgesia: A retrospective analysis of 7273 cases in a tertiary care teaching hospital. Eur J Anaesthesiol 2016;33:708-14.

24. El-Tahan MR. Role of Thoracic Epidural Analgesia for Thoracic Surgery and Its Perioperative Effects. J Cardiothorac Vasc Anesth 2017;31:1417-26.

25. Hill MV, Stucke RS, Billmeier SE, et al. Guideline for Discharge Opioid Prescriptions after Inpatient General Surgical Procedures. J Am Coll Surg 2018;226:996-1003.

26. Hill MV, Stucke RS, McMahon ML, et al. An Educational Intervention Decreases Opioid Prescribing After General Surgical Operations. Ann Surg 2018;267:468-72.

Cite this article as: Kodia K, Stephens-McDonnough JA, Alnajar A, Villamizar NR, Nguyen DM. Implementation of an enhanced recovery after thoracic surgery care pathway for thoracotomy patients-achieving better pain control with less (schedule II) opioid utilization. J Thorac Dis 2021;13(7):3948-3959. doi: $10.21037 /$ jtd-21-552
27. Kim MP, Chan EY, Meisenbach LM, et al. Enhanced recovery after thoracic surgery reduces discharge on highly dependent narcotics. J Thorac Dis 2018;10:984-90.

28. Brown LM, Kratz A, Verba S, et al. Pain and Opioid Use After Thoracic Surgery: Where We Are and Where We Need To Go. Ann Thorac Surg 2020;109:1638-45.

29. Katz J, Jackson M, Kavanagh BP, Sandler AN. Acute pain after thoracic surgery predicts long-term post-thoracotomy pain. Clin J Pain 1996;12:50-5.

30. Chen EY, Marcantonio A, Tornetta P. Correlation between 24-hour predischarge opioid use and amount of opioids prescribed at hospital discharge. JAMA Surg 2018;153:e174859. 
Supplementary

Table S1 Components of ERATS protocol at the University of Miami

Preoperative consultation

Extensive counseling of patients and family members about operative plans

Realistic expectation of postoperative recovery \& multimodal pain management

Printed information booklet with instructions

Preoperative clinic visit

Complete review of medical \& anesthesia history

Preoperative clearance

Routine preoperative instructions

2 bottles of carbohydrate drinks, 2 hours before surgery

Perioperative care

Acetaminophen $1,000 \mathrm{mg}$ (1 hour prior to surgery)

Gabapentin 100 mg (1 hour prior to surgery)

Prophylactic antibiotics (Cefazolin 2 gm for <120 kg or $3 \mathrm{gm}>120 \mathrm{~kg}$; Vancomycin 1,000 mg for penicillin allergy)

Anesthesia care: patient directed fluid management, anti-emetics

Intercostal nerve blocks and infiltration of surgical wounds with local anesthetics with liposomal bupivacaine

Postoperative care

Analgesics

Acetaminophen $1000 \mathrm{mg}$ per os (PO) Q (every) 8h

Tramadol 50 mg PO Q6h

Ibuprofen 600 mg PO Q8h post-operatively or Toradol 15 mg Q6h IV PRN for 2 days (if no medical contraindications) timing of first dose at the discretion of the attending surgeon

Gabapentin $100 \mathrm{mg}$ PO Q8h

Oxycodone 5 mg PO Q6h as needed (PRN) (pain scale: 4-6)

Oxycodone 10 mg PO Q6h PRN (pain scale: 7-10)

Morphine 2 to 4 mg IV Q6h PRN or Hydromorphone 0.5-1.0 mg IV or 2-4 mg p.o. Q6h PRN for breakthrough pain Heparin 5,000 U subcutaneous Q8h

Metoprolol $12.5 \mathrm{mg} \mathrm{Q12h} \mathrm{(if} \mathrm{not} \mathrm{already} \mathrm{on} \mathrm{a} \mathrm{beta-blocker} \mathrm{following} \mathrm{anatomic} \mathrm{resections)}$

Tamsulosin $0.4 \mathrm{mg}$ daily ( $>50$ years old)

Bowel regimen (Colace \& Dulcolax scheduled; Miralax \& Milk of magnesia PRN)

Incentive spirometer \& ambulation on POD 0

Regular diet on postoperative day 1

Assessment for home oxygen requirement (to prevent discharge delays)

Chest tube removal (postoperative day (POD) $1-2$, when volume $<5 \mathrm{~mL} / \mathrm{kg} /$ day)

Foley catheter removal (POD 2)

Intravenous fluid $1 \mathrm{~mL} / \mathrm{kg}$ until voiding

Discharge plan

Verbal \& printed discharge instructions

Contact ARNP or physician's office for advice and management of excessive neuropathic pain

Post-discharge analgesics

Acetaminophen 1,000 mg PO Q8 $\mathrm{h}$ for 20 days

Tramadol 50 mg PO Q6h for 3 days (12 tablets; if used postoperatively in-hospital)

Gabapentin 100 mg PO Q8 h for 60 days (30 days supply refill $\times 1$ ); titrating up to address post-discharge neurogenic pain

Ibuprofen $600 \mathrm{mg}$ PO Q8h for 20 days

Oxycodone 5 mg PO Q6 h PRN for 3 days (12 tablets; if used postoperatively in-hospital)

Pantoprazole 40 mg PO daily for 20 days

PO, per os; IV, intravenous; POD, post-operative day; PRN, as needed; Q, every; h, hour; ARNP, advanced registered nurse practioner. 